\title{
A Learning-Theory Approach to Sensor Networks
}

\author{
Supervised learning might be a viable approach to sensor network \\ applications. Preliminary research shows that a well-known algorithm \\ from learning theory effectively applies to environmental monitoring, \\ tracking of moving objects and plumes, and localization.
}

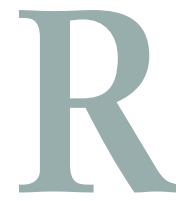
ecent advances in microelectromechanical systems, computing, and communication technology have sparked the emergence of massively distributed, wireless sensor networks with potentially thousands of nodes. Each node can sense the environment, process the collected data, and communicate with its peers or to an external observer. A steady increase in these networks' capabilities and decrease in the cost of producing them have made possible applications that seemed too expensive or unrealistic. ${ }^{1}$ On the other hand, these changes create great theoretical challenges in distributed signal processing, control, and other areas.

My colleagues and I in the Department of Electrical Engineering and Computer Sciences at the

Slobodan N. Simić University of California, Berkeley University of California, Berkeley, propose a unified approach to various sensor network applications, using supervised learning. Supervised learning refers to learning from examples, in the form of input-output pairs, by which a system that isn't programmed in advance can estimate an unknown function and predict its values for inputs outside the training set. In particular, we examined random wireless sensor networks, in which nodes are randomly distributed in the region of deployment. When operating normally, nodes communicate and collaborate only with other nearby nodes (within communication range). However, a base station-with a more powerful computer on board-can query a node or group of nodes when necessary and perform data fusion. We mainly considered the sensor network platform developed at UC Berkeley (http:// webs.cs.berkeley.edu), but our approach applies to a broader framework.

The problem of learning from data becomes crucial in a world flooded with too much information. Examples of this abound in biology, particle physics, astronomy, engineering, and other disciplines. Instrumenting the physical world with wireless sensors will generate massive amounts of data (examples) that can be processed (learned from) efficiently and usefully. This is where learning theory comes into play.

Learning techniques have been applied in many diverse scenarios. We considered some basic concepts of learning theory and how they might address the needs of random wireless sensor networks. Our research is still in progress, so our ideas and results are preliminary; however, we offer a beneficial perspective for the sensor network community to consider.

\section{Basic notions of learning theory}

The work of Tomaso Poggio, ${ }^{2}$ Steve Smale, ${ }^{2,3}$ and Felipe Cucker ${ }^{3}$ was important to our understanding of learning theory. Vladimir Vapnik's fundamental work ${ }^{4}$ is also an excellent resource on statistical learning.

In supervised learning, input-output pairs represent a sample of the object (such as a function) to be learned. To illustrate how systems are trained with these, consider the classic example of learning a physical law by curve fitting to data. 
Suppose that the physical law to be learned is an unknown function $f: \mathbb{R} \rightarrow \mathbb{R}$ $(\mathbb{R}$ denotes the set of real numbers) of a special form. Assume, for instance, that $f$ is a polynomial of degree $N$, with coefficients $w_{0}, \ldots, w_{N}$. Let $\left(x_{1}, y_{1}\right), \ldots$, $\left(x_{m}, y_{m}\right)(m>N)$ be samples of $f$, where we obtain $y_{i}$ by "measuring" $f$ at $x_{i}$. If the measurements were exact, we would have $y_{i}=f\left(x_{i}\right)$, but in reality we expect them to be affected by noise. Learning $f$ amounts to finding the vector of coefficients $w=\left(w_{0}, \ldots, w_{N}\right)$ such that

$$
f_{w}(x)=\sum_{i=0}^{N} w_{i} x^{i}
$$

is the best fit for the data. We can do this efficiently by the Gaussian method of least squares, which computes $w$ to minimize

$$
\sum_{i=1}^{m}\left(f_{w}\left(x_{i}\right)-y_{i}\right)^{2}
$$

Often (such as in the context of random wireless sensor networks), the $x_{i}$ are random rather than chosen.

In general, we are given a set of data $\left(x_{1}, y_{1}\right), \ldots,\left(x_{m}, y_{m}\right)$, where the $x_{i}$ 's are in some input space $X$, usually a closed subset of some $\mathbb{R}^{k}$, and the $y_{i}$ 's belong to an output space $Y$, which we assume to be $\mathbb{R}$. For data generated by a sensor network, $x_{i}$ is the position of the $i$ th sensor node and $y_{i}$ is its measurement or some function of it. I'll explain this in more detail later.

On the space $X \times Y$ consisting of all input-output pairs $(x, y)$, with $x \in X$ and $y \in Y$, we assume a probability measure $\rho$ exists that governs the sampling. ${ }^{5}$ That is, pairs $\left(x_{i}, y_{i}\right)$ are randomly picked according to $\rho$. The measure $\rho$ defines a function $f_{\rho}: X \rightarrow Y$, called the regression function of $\rho$. For each input $x, f_{\rho}(x)$ is the average (with respect to $\rho$ ) value of the output $y$ in $\{(x, y): y \in Y\}$. The goal is then to learn (that is, find a good approximation of) $f_{\rho}$ from random samples $\left(x_{1}, y_{1}\right), \ldots,\left(x_{m}, y_{m}\right)$.

In other words, we'd like to find a function $f: X \rightarrow Y$ that minimizes the error

$$
\int_{X}\left(f-f_{\rho}\right)^{2} d \rho_{X}
$$

where $\rho_{X}$ is the marginal measure on $X$ induced by $\rho .^{5}$

The central question of learning theory is how well f generalizes - that is, "how well it estimates the outputs for previously unseen inputs." 2

\section{The key algorithm}

We use an algorithm-the key algorithm from the works mentioned earlier ${ }^{2,3}$ - that fits the training data set $\left(x_{1}, y_{1}\right), \ldots,\left(x_{m}, y_{m}\right) \in X \times Y$ with a function $f: X \rightarrow Y$, where $X$ is a closed subset of some $\mathbb{R}^{k}$ and $Y=\mathbb{R}$.

A function $K: X \times X \rightarrow \mathbb{R}$ is symmetric if $K\left(x, x^{\prime}\right)=K\left(x^{\prime}, x\right)$, for all $x, x^{\prime} \in X$. It's positive definite if, in addition, for any finite subset $\left\{p_{1}, \ldots, p_{n}\right\}$ of $X$, the $n \times n$ matrix with entries $K\left(p_{i}, p_{j}\right)$ is positive definite. This means that for all real numbers $c_{1}, \ldots, c_{n}$,

$$
\sum_{i, j} c_{i} c_{j} K\left(p_{i}, p_{j}\right) \geq 0 .
$$

If $K$ is symmetric, positive definite, and continuous, we call it a Mercer kernel. Examples are

$$
K\left(x, x^{\prime}\right)=\exp \left(-\frac{\left\|x-x^{\prime}\right\|^{2}}{2 \sigma^{2}}\right)
$$

and

$$
K\left(x, x^{\prime}\right)=\left(a^{2}+\left\|x-x^{\prime}\right\|^{2}\right)^{-\alpha},
$$

where $\|\cdot\|$ denotes the 2 -norm and $a, \alpha$, $\sigma>0$.

The algorithm is as follows:
1. Start with data $\left(x_{1}, y_{1}\right), \ldots,\left(x_{m}, y_{m}\right)$. 2. Choose a Mercer kernel such as the one in Equation 1.

3. Choose a positive real number $\gamma$ and let $\mathbf{c}=\left(\mathrm{c}_{1}, \ldots, \mathrm{c}_{m}\right)$ be the unique solution to the equation

$(m \gamma \mathbf{I}+\mathbf{K}) \mathbf{c}=\mathbf{y}$

where $\mathbf{I}$ is the $m \times m$ identity matrix, $\mathrm{K}$ is the square positive-definite matrix with entries $K\left(x_{i}, x_{j}\right), 1 \leq i, j$ $\leq m$, and $y$ is the column vector with components $y_{1}, \ldots, y_{m}$.

4. Define $f: X \rightarrow Y$ by

$$
f(x)=\sum_{i=1}^{m} c_{i} K\left(x_{i}, x\right) .
$$

Because the matrix $m \gamma \mathbf{I}+\mathbf{K}$ is positive definite, and therefore nonsingular, Equation 2 is well posed.

So what does the approximating function $f$ look like? If the kernel is Gaussian (see Equation 1), $f$ is a weighted sum of Gaussian blobs, each centered at one of the $x_{i}$ 's.

The weights $c_{i}$ minimize the error on the training set. The parameter $\sigma$ from Equation 1 controls the degree of smoothing, noise tolerance, and generalization. For instance, as $\sigma$ approaches $0, f$ becomes just a "look-up table" that gives the exact value of $y$ when $x=x_{i}$ but does not generalize to other values of the input. The parameter $\gamma$ ensures that Equation 2 is well posed: the larger the $m \gamma$, the better the condition number of Equation 2. On the other hand, if $\gamma$ were zero, $f\left(x_{i}\right)$ would equal $y_{i}$ exactly. It turns out that a best $\gamma$ exists that solves this trade-off problem. ${ }^{6}$

This algorithm has been used in many problems. It performs well in a variety of applications involving regression as well as binary classification. Systems like Equation 2 have been studied since Gauss's time; algorithms to efficiently 


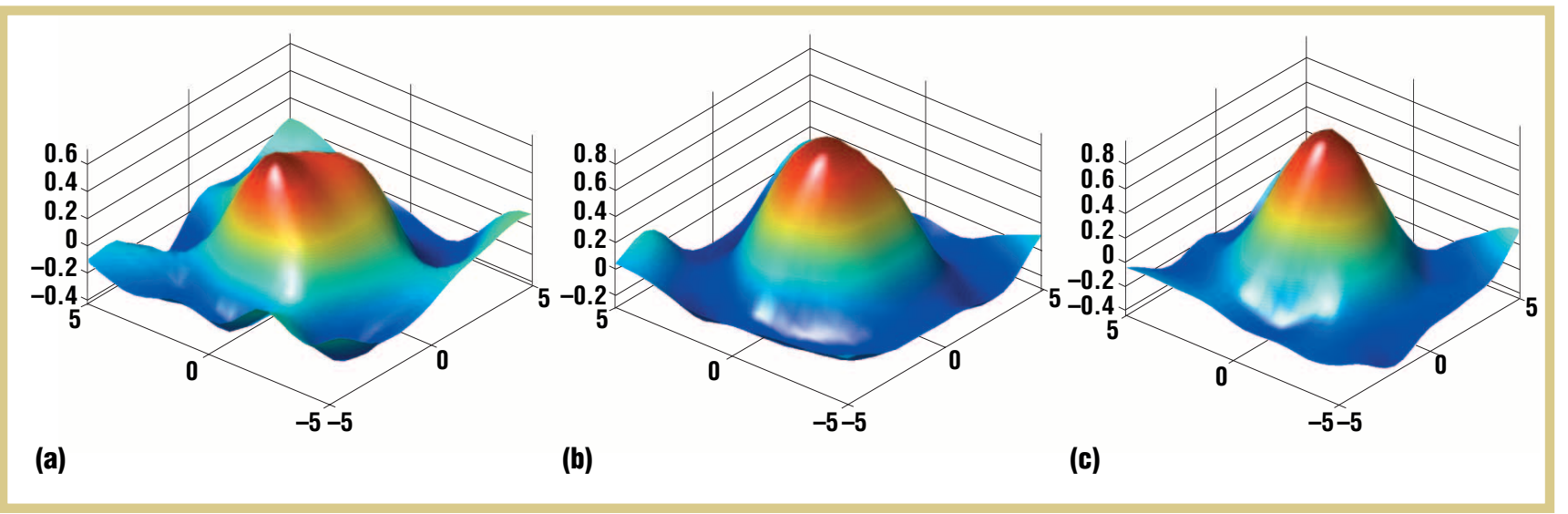

Figure 1. Estimates of $f_{0}$ for (a) $m=100$, (b) $m=200$, and (c) $m=500$ nodes, when $\gamma=0.001, \Sigma^{2}=0.01$, and $\sigma^{2}=2$.

compute their solutions form one of the most developed fields of numerical analysis. $^{2}$

To illustrate the algorithm, let's apply it to a training set of $m$ points $\left(x_{i}, y_{i}\right)$, where the inputs $x_{i}$ are random, generated by the uniform distribution on the square $Q=$ $[-5,5]^{2}$. The output $y_{i}$ is a Gaussian random variable with mean $f_{0}\left(x_{i}\right)$ and variance $\Sigma^{2}$, where $f_{0}(x)=\sin \|x\| /\|x\|$. We want to estimate the (unknown) function $f_{0}$ given noisy data. Figure 1 shows how the graph of the estimate changes as $m=100$, 200, and 500. Figure 2 illustrates how the estimate changes as $\gamma$ varies.

\section{Sensor network applications}

Most applications to sensor networks can be formulated as learning about the environment through examples. Usually, we're interested in a particular feature of the environment that can be represented by a vector-valued function defined on the region in which our sensor network is deployed. Let $X$ be that region. If a sensor network is deployed on the ground, we can take $X$ to be a closed subset of $\mathbb{R}^{2}$. If the region is significantly curved (for example, mountainous), we can take $X$ to be a $2 \mathrm{D}$ surface in $\mathbb{R}^{3}$. If the sensor network is airborne or deployed in water, we can take $X \subset \mathbb{R}^{3}$.

Data that the sensor network generates are of the desired form $\left(x_{1}, y_{1}\right), \ldots$, $\left(x_{m}, y_{m}\right)$, where $x_{i} \in X$ is usually the (estimated) location of the $i$ th sensor node and $y_{i}$ is an $\ell$-tuple of real numbers rep- resenting measurements of the $i$ th node. Because the framework from the earlier section requires the outputs to be scalar, we decompose each $y_{i}$ into scalar components $y_{i}=\left(y_{i}^{1}, \ldots, y_{i}^{\ell}\right)$ and work with data sets $\left(x_{i}, y_{i}^{j}\right)_{i=1}^{m}$, for $j=1, \ldots, \ell$, separately. So, without loss, we can assume that our sensor network outputs are scalar.

Recall that points $\left(x_{i}, y_{i}\right)$ are picked from $X \times Y$ according to some unknown probability measure $\rho$, which represents the noise in the measurements. What we'd like to estimate (or learn) is the regression function $f_{\rho}$ of $\rho$, which in our context is some feature of the environment represented by the outputs $y_{i} \approx f_{\rho}\left(x_{i}\right)$.

Applying the algorithm to sensor networks involves these problems:

Figure 2. Estimates of $f_{0}$ for (a) $\gamma=0.1$, (b) $\gamma=0.01$, and (c) $\gamma=0.001$, when $m=100$ nodes, $\Sigma^{2}=0.01$, and $\sigma^{2}=2$.

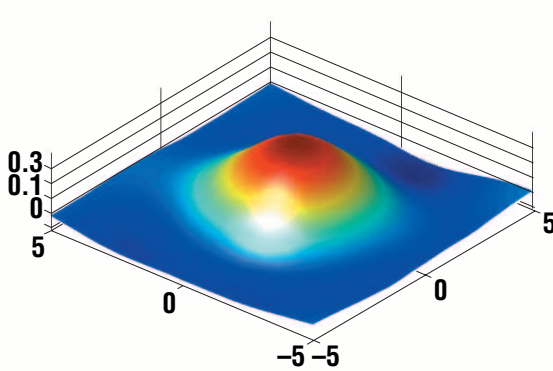

(a)

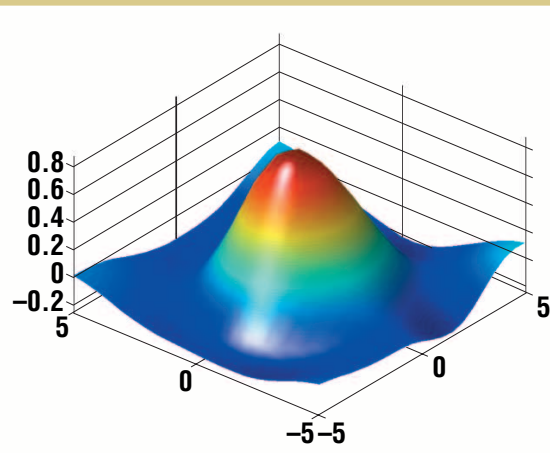

(b)

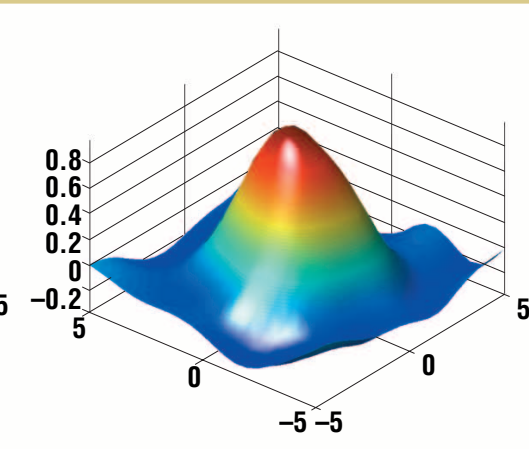

(c) 
- How much computation should be distributed to individual nodes, and how much of it should be centralized? - Which kernel should we choose?

- Which values of $\gamma$ and $\sigma$ are best?

This article discusses only the first problem; the latter two are beyond its scope. (See Cucker and Smale's article ${ }^{6}$ about finding the best $\gamma$.)

Centralized computation might require much long-range communication with a base station, which consumes much energy but might allow for more intensive data processing by a more powerful base station computer. On the other hand, distributed-or localizedcomputation at each node means less long-range communication, but the amount of computation possible will probably be significantly limited. This is the usual trade-off between communication and computation in sensor networks. However, because transmitting a bit of information is still an order of magnitude more expensive than computing it, we opt for a more distributed alternative, assuming that nodes can do a nontrivial amount of computation (such as solving reasonably large systems of linear equations).

We can summarize our general approach, which we can call distributed sensorweb learning, as follows: Each node $S$ generates its data set by communicating with its neighbors and applies the algorithm to the set to obtain its own estimate $f_{S}$.

We call a node a neighbor of a node $S$ if it can establish a bidirectional communication link with $S$. Denote by $D_{i}$ the communication region (or neighborhood) of node $S_{i}(i=1, \ldots, m)$-that is, the subset of $X$ such that all nodes in $D_{i}$ are $S_{i}$ 's neighbors. We'll assume that $D_{i}$ is the disk centered at $x_{i}$ (the position of $S_{i}$ ) of radius $r$ (the communication range). The estimate $f_{i}$ by $S_{i}$ of an unknown function $f$ is then valid only in $D_{i}$. What happens if the

communication neighborhoods of two nodes $S_{i}$ and $S_{j}$ overlap? Which estimate do we accept in the intersection $D_{i} \cap D_{j}$ ? That is, if the need arises, how should the base station merge the estimates $f_{i}$ and $f_{j}$ ? We have a preliminary solution that glues the local estimates by taking a certain type of average.

Denote by $A_{i}$ and $B_{i}$ the disks of radius $r / 2$ and $3 r / 4$, respectively, centered at the $i$ th node $S_{i}$. Let $\beta_{i}: i=1, \ldots, m$ be a col-

\section{Distributed computation at each node}

\section{means less long-range communication, but the}

\section{amount of computation possible will probably}

\section{be significantly limited.}

lection of real-valued functions defined on the whole region of deployment $X$ and satisfying these properties:

- The $\beta_{i}$ 's are smooth

- $0 \leq \beta_{i} \leq 1, \sum_{i} \beta_{i}(x)=1$ at every point $x \in X$

- $\beta_{i}=1$ on $A_{i}$

- $\beta_{i}=0$ outside $B_{i}$

A collection such as this exists, as is well known in differential geometry, and is called a partition of unity. Each $\beta_{i}$ is what's often called a bump function because its graph looks like a bump. Let $f_{i}$ be the local estimate of an unknown function $f$, obtained at the $i$ th node using the distributed algorithm; it's defined only on the set $D_{i}$ the communication region of the $i$ th node. Extend $f_{i}$ to the function $\bar{f}_{i}$ defined on all of $X$ by defining $\bar{f}_{i}$ to be equal to $f_{i}$ on $D_{i}$ and zero outside $D_{i}$. We glue the $\bar{f}_{i}$ 's into global estimate $f_{*}$ by setting

$$
f_{*}=\sum_{i=1}^{m} \beta_{i} \bar{f}_{i}
$$

dent, uniformly distributed random variables on $X$, but other distributions are possible in particular situations.

The following examples suggest how to formulate particular sensor network applications from a supervised-learning viewpoint.

\section{Environmental monitoring}

In this case, we want to estimate some unknown scalar environmental function $f_{\text {env }}$ defined on $X$, such as temperature, air pressure, humidity, or light. ${ }^{7}$ We can assume that the output of the measurement $y_{i}$ at the node $S_{i}$ is a normally distributed random variable with mean $f_{\text {env }}\left(x_{i}\right)$. When we apply the distributed algorithm, each node gets a local estimate of $f_{\text {env }}$. We can find upper bounds for the local error with a desired confidence by using the results of Cucker and Smale's work; ${ }^{3}$ they'll depend on the number of neighbors each node has and on other network parameters.

We might also want to estimate a vector field $F$, such as the gradient of temperature, representing the heat flow in the environment. ${ }^{8}$ We can do this by reducing the task into estimating the 


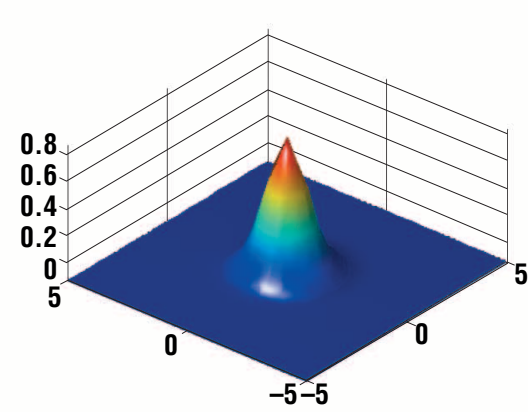

(a)

Figure 3. Temperature graphs: (a) The true temperature and (b) an estimate with $m=$ 100 nodes.

field's scalar components by each node.

\section{Plume tracking}

Suppose we want to track a plume of hazardous gas moving slowly through region $X .{ }^{9,10}$ Let $A$ be the set representing the plume's extent at a time instant $t_{0}$. Let $f_{A}$ be $A$ 's indicator function - that is, $f_{A}(x)=1$ if $x \in A$ and $f_{A}(x)=0$ otherwise. Estimating $A$ amounts to estimat$\operatorname{ing} f_{A}$. Each node $S_{i}$ measures the concentration of the gas at its location and outputs $y_{i}=1$ if the concentration is larger than some threshold $\tau$; otherwise $y_{i}=0$.

Alternatively, $S_{i}$ can output its numerical measurement and the network can estimate the concentration function

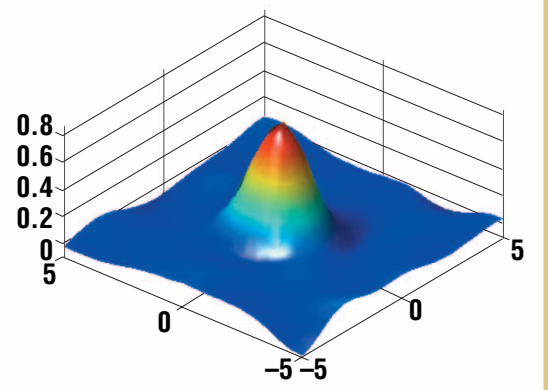

(b)

amounts to estimating the positions of objects in $\mathcal{O}$ with accuracy $\delta$. Once again, the inputs $x_{i}$ are positions of sensor nodes and the outputs $y_{i}$ are +1 (if at least one element of $\mathcal{O}$ is within the distance $\delta$ from $S_{i}$ ) or 0 (otherwise).

\section{Localization}

We can approach node localization similarly. Suppose a certain number of nodes (for example, beacons) know their positions with some accuracy. Often the sensor network's first task is to localize (that is, estimate the positions of) the remaining nodes. ${ }^{11,12}$ Let $S$ be a node with unknown position $p \in X$ and let $\delta>0$ be the desired accuracy. Then $S$ can communicate with the neighbors who know their positions and gather data $\left(x_{i}, y_{i}\right) \cdot x_{i}$ is the position of a known neighbor and $y_{i}=1$ if the distance between that neighbor and $S$ is less than $\delta$ and 0 otherwise. Learning from the given data, $S$ gets an estimate of the indicator function of the disk centered at $p$ with radius $\delta$. We can take the point where the estimate reaches its global maximum to be $S$ 's position.

\section{Simulation results}

Following are some preliminary simulation results in Matlab. We used the centralized version of the algorithm with a Gaussian kernel (See Equation 1).

Figure 3 compares the graphs of the true temperature function $T(x)$ for $x \in$ $[-5,5]^{2}$ (Figure $\left.3 \mathrm{a}\right)$ and its estimate (Figure $3 b)$. We took $T(x)=\exp \left(-\|x\|^{2}\right)$ and assumed the output $y_{i}$ is a Gaussian random variable with mean $T\left(x_{i}\right)$ and variance 0.001 . The number of nodes is $m=$ $100, \sigma^{2}=0.5$, and $\gamma=0.0001$.

Figure 4 shows two estimates of the set $\mathrm{A}=\{(u, v):|v|<1\}$ (which you can consider as being occupied by a plume of gas), using $m=200$ and 400 nodes, with $\sigma^{2}=2$ and $\gamma=0.001$. Pictured are the graphs of the estimates of $A$ 's indicator function. 
Figure 5 shows a localization estimate with $\sigma^{2}=2, \gamma=0.0001$, and $\delta=0.9$. We assumed the unknown position of node $S$ is $(0,0) ; S$ has $m$ neighbors $(m=30$ and 60), each aware of its own position. The figure shows the graph of the function estimating the indicator function of the disk of radius $d$ centered at the origin. The point where it achieves its maximum (ideally, 1) can be taken as the estimate of $S$ 's position.

upervised learning theory offers an effective approach to sensor networks. We've demonstrated this through showing how a wellknown learning algorithm can be used in the context of environmental monitoring, tracking, and localization. In the future-especially in dense, large-scale sensor networks-we foresee even greater possibilities of such applications, which we plan to investigate in future work. $\mathbb{P}$

\section{ACKNOWLEDGMENTS}

NSF grant EIA-0122599 partly supported our research.

\section{REFERENCES}

1. D. Estrin et al., "Connecting the Physical World with Pervasive Networks," IEEE Pervasive Computing, vol. 1, no. 1, Jan.Mar. 2002, pp. 59-69.

2. T. Poggio and S. Smale, "The Mathematics of Learning: Dealing with Data," Notices Am. Math. Soc., vol. 50, no. 5, May 2003, pp. 537-544.

3. F. Cucker and S. Smale, "On the Mathematical Foundations of Learning," Bull. Am. Math. Soc., vol. 39, no. 1, Oct. 2001, pp. $1-49$.

4. V. Vapnik, Statistical Learning Theory, John Wiley \& Sons, 1998.

5. G.R. Grimmett and D.R. Strizaker, Probability and Random Processes, Oxford Univ. Press, 1997.

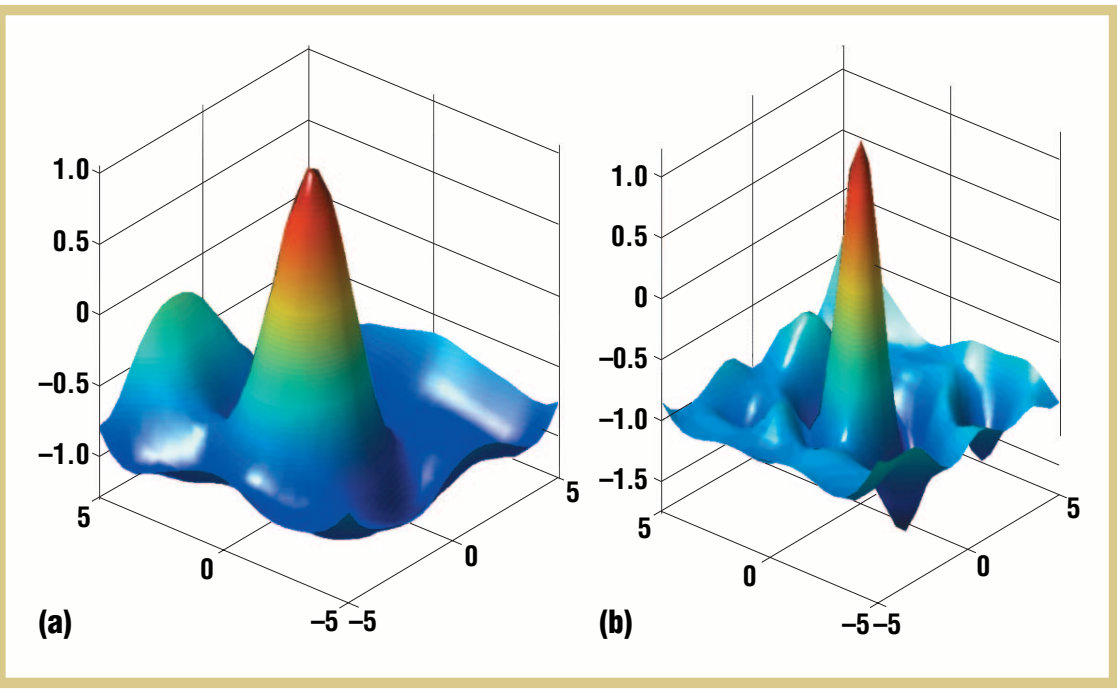

Figure 5. A position estimate using (a) $m=30$ and (b) $m=60$ nodes in $[-5,5]^{2}$, when $\sigma^{2}=2, \gamma=0.0001$, and $\delta=0.9$. The correct position is at $(0,0)$.

6. F. Cucker and S. Smale, "Best Choices for Regularization Parameters in Learning Theory: On the Bias-Variance Problem," Foundations of Computational Math., vol. 2, no. 4, Springer-Verlag, Oct. 2002, pp. 413-428.

7. A. Mainwaring et al., "Wireless Sensor Networks for Habitat Monitoring," Proc. 1st ACM Int'l Workshop Wireless Sensor Networks and Applications, ACM Press, 2002, pp. 88-97.

8. S.N. Simić and S. Sastry, "Distributed Environmental Monitoring Using Random Sensor Networks," Proc. 2nd Int'l Workshop Information Processing in Sensor Networks (IPSN 2003), LNCS 2634, Springer-Verlag, 2003, pp. 582-592.

9. K.K. Chintalapudi and R. Govindan, Localized Edge Detection in Sensor Fields, tech. report 02-773, Univ. Southern California, 2002; http://cs.usc.edu/ ramesh/papers/ edge_detection.pdf.
10. R. Nowak and U. Mitra, "Boundary Estimation in Sensor Networks: Theory and Methods," Proc. 2nd Int'l Workshop Information Processing in Sensor Networks (IPSN 2003), LNCS 2634, SpringerVerlag, 2003, pp. 80-95.

11. S.N. Simić and S. Sastry, "A Distributed Algorithm for Localization in Random Wireless Networks," to be published in Discrete Applied Mathematics, Elsevier.

12. S.N. Simić and S. Sastry, Distributed Localization in Wireless Ad Hoc Networks, tech. memo UCB/ERL M02/26, Dept. of Electrical Eng. and Computer Sciences, Univ. California, Berkeley, 2002.

For more information on this or any other computing topic, please visit our Digital Library at http:// computer.org/publications/dlib.

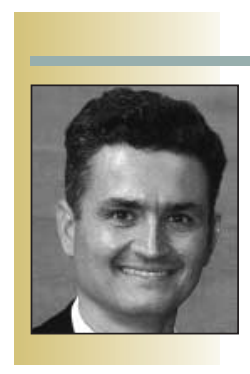

the AUTHOR 